\title{
Zonally Magnified Oblique Multislice and Non-Zonally Magnified Oblique Multislice DWI of the Cervical Spinal Cord
}

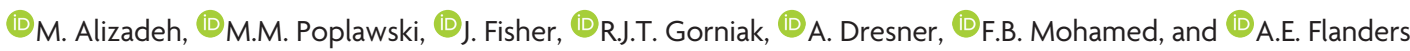

\begin{abstract}
BACKGROUND AND PURPOSE: The zonally magnified oblique multislice EPI (ZOOM-EPI) diffusion-weighted sequence has been visually shown to provide superior MR diffusion image quality compared with the full-FOV single-shot EPI sequence (non-ZOOM-EPI) in the adult cervical spinal cord. The purpose of this study was to examine the diffusion tensor imaging indices in the normal human cervical spinal cord between ZOOMED and non-ZOOMED DTI acquisitions and determine whether DTI values are comparable between direct and indirect age-matched groups.
\end{abstract}

MATERIALS AND METHODS: Fifty-four subjects 23-58 years of age ( 9 direct age-matched and 45 indirect age-matched) were scanned using a 1.5T scanner. Diffusion tensor indices including fractional anisotropy, mean diffusivity, axial diffusivity, and radial diffusivity were generated from the DTI dataset. These DTI values were calculated for both ZOOM and non-ZOOM acquisitions and compared at each intervertebral disc level. The variability of the DTI values for ZOOM and non-ZOOM sequences was measured using a coefficient of variation within direct and indirect age-matched controls.

RESULTS: The mean diffusivity, axial diffusivity, and radial diffusivity values obtained along the cervical spinal cord in the age-matched controls showed a significant decrease using the ZOOM sequence $(P=.05, P=.002$, and $P<.001)$. Mean fractional anisotropy showed a significant increase $(P=.04)$ using the ZOOM sequence. The indirect age-matched controls showed a statistically significant increase in fractional anisotropy $(P=.03)$ and a decrease in mean diffusivity $(P=.002)$, axial diffusivity $(P<.001)$, and radial diffusivity $(P=.002)$ using the ZOOM sequence. Less variability has been shown in DTI using the ZOOM sequence compared with the non-ZOOM sequence in both direct and indirect age groups. The ZOOM sequence exhibited higher SNR (SNR $\mathrm{ZOOM}=22.84 \pm 7.59)$ compared with the non-ZOOM sequence $\left(S_{N R}\right.$ non-ZOOM $\left.=19.7 \pm 7.05\right)$. However, when we used a 2-tailed $t$ test assuming unequal variances, the ZOOM sequence did not demonstrate a statistically significant increase.

CONCLUSIONS: ZOOM DTI acquisition methods provide superior image quality and precision over non-ZOOM techniques and are recommended over conventional full-FOV single-shot EPI DTI for clinical applications in cervical spinal cord imaging.

ABBREVIATIONS: $A D=$ axial diffusivity; $\mathrm{CoV}=$ coefficient of variation; $\mathrm{FA}=$ fractional anisotropy $\mathrm{MD}=$ mean diffusivity; $\mathrm{RD}=$ radial diffusivity; $\mathrm{ZOOM}=$ zonally magnified oblique multislice

I: n recent years, techniques like diffusion tensor imaging of the spinal cord have become powerful quantitative tools that track the diffusion of water molecules throughout the length of the cord. ${ }^{1}$ DTI has been used in past studies to investigate white matter and its axonal projections in the cord. However, it is difficult to

Received February 18, 2018; accepted after revision April 26.

From the Department of Radiology (M.A., M.M.P., J.F., R.J.T.G., A.D., F.B.M., A.E.F.), Jefferson Integrated Magnetic Resonance Imaging Center, and Department of Neurosurgery (M.A.), Thomas Jefferson University, Philadelphia, Pennsylvania.

Please address correspondence to Adam E. Flanders, MD, 909 Walnut St, Jefferson Integrated Magnetic Resonance Imaging Center, Department of Radiology, Thomas Jefferson University, Philadelphia, PA 19107; e-mail:

Adam.Flanders@jefferson.edu

http://dx.doi.org/10.3174/ajnr.A5703 obtain distortion-free, high-resolution diffusion-weighted images of the spinal cord using single-shot EPI. ${ }^{1}$ Long readout times and low bandwidths in the phase-encode direction of the standard single-shot EPI sequence increase the possibility of distortion in the resultant images. ${ }^{1}$ Coherent macroscopic or bulk motion varies during each echo acquisition; this variation can give rise to ghosting artifacts in the final images. Motion adds additional phase to the spins, which will also challenge the phase requirements between transverse magnetization and radiofrequency pulses. ${ }^{1,2}$ Despite these challenges, single-shot EPI offers a short acquisition time that decreases the probability of motionrelated artifacts, making this technique most appealing for clinical imaging of the spinal cord. ${ }^{3}$

Recently, reduced FOV imaging sequences have generated 
valuable spinal cord imaging data. Diffusion-weighted imaging sequences like the zonally magnified oblique multislice (ZOOM) sequence have used this reduced FOV to acquire diffusion metrics in the spinal cord. ${ }^{3,4}$ The impetus was to create a ZOOM acquisition that could replace the standard full-FOV method for clinical use. This is predicated on the goal of keeping acquisition times as close to or less than those of conventional full-field DTI techniques, with superior results. Several reduced FOV diffusionweighted sequences, including the ZOOM sequence and the inner FOV 2D radiofrequency excitation and overvolume suppression techniques, have been applied to obtain DTI of the spinal cord. It has been shown that in nearly all these instances, the reduced FOV EPI DTI method provides an increase in the signal-to-noise ratio compared with conventional full-FOV EPI techniques (nonZOOM). ${ }^{3,5,6}$ Using these techniques, several researchers have been able to acquire more precise diffusion metrics using ZOOM DTI sequences. In 1 study, ZOOM DTI increased diagnostic confidence in $33 \%$ of clinical examinations in a sample size of 180 clinical scans. ${ }^{7}$ This cohort included a range of various pathologies such as demyelination, acute infarction, infection, and abnormal tissue growth. ${ }^{7}$ Other groups have used these techniques to investigate the healthy population, ranging from mapping of the optic nerve, ${ }^{8}$ assessing the pediatric spinal cord, and comparing of intrinsic artifacts among imaging techniques. ${ }^{9}$ In each use, the ZOOM DTI sequences have been shown useful for clinical application.

The purpose of this study was to perform a quantitative comparison of ZOOM and non-ZOOM DTI techniques in the adult cervical spinal cord. We hypothesized that due to the inherent capability of ZOOM to reduce susceptibility artifacts coupled with a higher SNR, the DTI metrics generated from ZOOM methods would be more precise. A direct age match compares data from the same subject scanned using both imaging sequences, while an indirect age match is a comparison of 2 different subjects whose ages match. In theory, a direct age match will reduce variation that may arise from natural anatomic and diffusive differences among participating subjects compared with indirect agematched groups.

\section{MATERIALS AND METHODS \\ Subject Recruitment}

In this prospective monocenter study, 54 patients between 23 and 58 years of age (mean age, $38.09 \pm 8.5$ years; 26 men and 28 women) were recruited. Each of the patients was deemed healthy; with no clinical or imaging criteria of spinal cord pathology. Examinations were performed as part of clinical care for screening in trauma to exclude soft-tissue injuries. Nine of the recruited subjects underwent scans that implemented both acquisition techniques that were used for a direct age-matched comparison. In addition, an indirect age-matched group was created using a separate group of 22 subjects who were scanned using the nonZOOM technique and who were later used to compare with the 23 subjects scanned using the ZOOM technique. The diffusion data of this study were compared using the 2 different methods. The study was approved by the institutional review board of Thomas Jefferson University Hospital. Informed consent was obtained from all patients.

\section{Image Acquisition}

All MR imaging scans were performed on the same magnet using an Achieva 1.5T scanner (Philips Healthcare, Best, Netherlands) with a 16-channel neurovascular array coil, including 8 head elements and 4 neck and 4 chest elements. Axial DTI covering the cervical spinal cord was acquired using either the ZOOM or nonZOOM techniques in the same anatomic location prescribed for the T2-weighted images to cover the entire cervical spinal cord (C1 to C7-T1 levels). The non-ZOOM DTI scan parameters were the following: $\mathrm{TR}=6176 \mathrm{~ms}, \mathrm{TE}=82 \mathrm{~ms}$, number of signal averages $=8, \mathrm{FOV}=220 \times 130 \mathrm{~mm}$, matrix $=144 \times 84$, number of slices $=36$, slice thickness $=4 \mathrm{~mm}$, voxel size $=1.53 \times 1.53 \times$ $4.0 \mathrm{~mm}$. The ZOOM scan parameters were the following: $\mathrm{TR}=$ $4950 \mathrm{~ms}, \mathrm{TE}=95 \mathrm{~ms}$, number of signal averages $=4$, FOV $=$ $110 \times 110 \mathrm{~mm}$, matrix $=96 \times 96$, number of slices $=32$, slice thickness $=4 \mathrm{~mm}$, voxel size $=1.15 \times 1.15 \times 4.0 \mathrm{~mm}$. Both sequences used 6 directions at $b=800 \mathrm{~s} / \mathrm{mm}^{2}$. The acquisition time of each technique was kept under 5 minutes. The imaging acquisition time was deliberately minimized to make it as clinically practical as possible; therefore, neither gating nor anesthesia was used during scanning.

In both techniques, manual shim and fat saturation volume adjustments were also performed before data acquisition to confine the adjustment volume to the anatomy of interest as much as possible to limit residual distortions and chemical shift artifacts.

\section{Image Processing}

Initially, DTI volumes were corrected for motion-induced artifacts by aligning diffusion directional images to the reference image $\left(B_{0}\right)$ based on the affine transformation technique. Next, DTI scalars, including fractional anisotropy (FA), mean diffusivity $(\mathrm{MD})$, axial diffusivity (AD), and radial difusivity (RD), were computed using a linear estimation of the tensor in DTI FiberTrak software (Philips Healthcare; https://www.usa.philips.com/ healthcare/product/HCNMRB617/dti-fibertrak-mr-software). Global thresholding and smoothing techniques were applied to remove background, decrease the computational time, and minimize the effects of noise in the tensor calculation.

\section{Manual ROI Definition}

To obtain DTI metrics, we manually drew ROIs at each cervical intervertebral disc level on the generated FA map in the axial plane. These intervertebral disc levels included $\mathrm{C} 1-\mathrm{C} 2, \mathrm{C} 2-\mathrm{C} 3$, C3-C4, C4-C5, C5-C6, C6-C7, C7-T1. Each ROI was drawn within 1-2 voxels of the cord boundary to prevent partial volume effects from the nearby cord/CSF junction. ${ }^{3}$ Figure 1 shows the mean size of the ROIs at each intervertebral disc level in all subjects.

\section{Statistics}

On the basis of the definition of whole-cord ROIs, we performed statistical analysis between the non-ZOOM and ZOOM groups. A comprehensive data table was created containing information about DTI measures (Tables 1 and 2). Means and SDs of each measure for every subject along the cervical spinal cord were calculated. These measures then were compared between nonZOOM and ZOOM subjects for both indirect and direct groups 


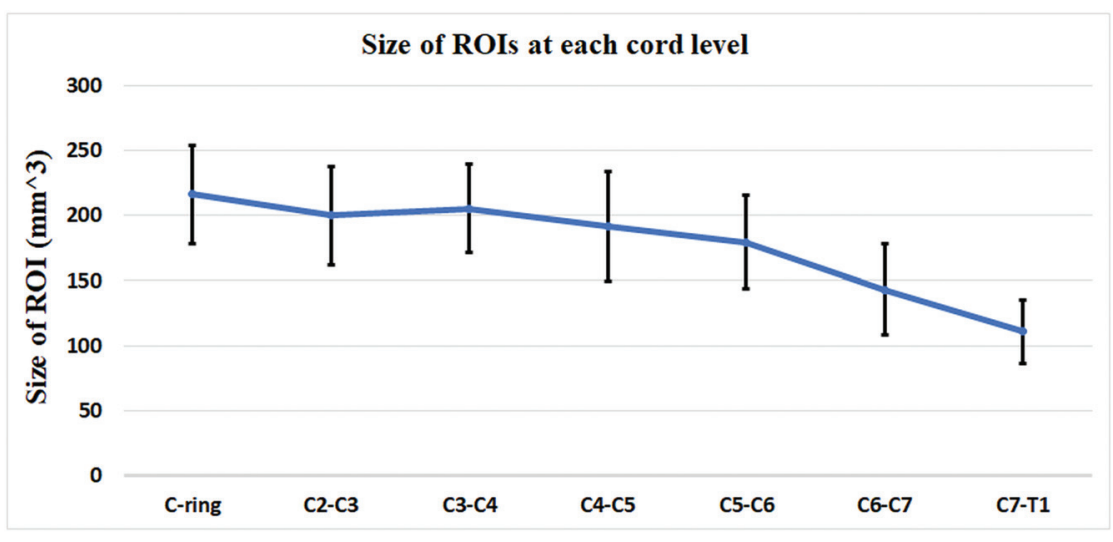

FIG 1. The size of ROIs manually drawn at each intervertebral disc level. The error bars represent the SDs.

\begin{tabular}{|c|c|c|c|}
\hline & Non-ZOOM & ZOOM & Prob $<|t|$ \\
\hline FA & $0.65 \pm 0.042$ & $0.74 \pm 0.038$ & $P=.04$ \\
\hline MD & $0.72 \pm 0.038$ & $0.50 \pm 0.032$ & $P=005$ \\
\hline$A D$ & $1.36 \pm 0.086$ & $0.99 \pm 0.021$ & $P=.002$ \\
\hline RD & $0.42 \pm 0.041$ & $0.24 \pm 0.037$ & $P<.001$ \\
\hline
\end{tabular}

Note: - Prob $<|t|$ indicates the $P$ value for 2-tailed test.

${ }^{\mathrm{a}}$ Data are means and SDs.

Table 2: Comparison of non-ZOOM and ZOOM of averaged FA, $M D, A D$, and $R D$ values along the cervical spinal cord levels and across indirect age-matched subjects ${ }^{\mathrm{a}}$

\begin{tabular}{lccl}
\hline & Non-ZOOM & ZOOM & Prob<|t| \\
\hline FA & $0.65 \pm 0.067$ & $0.76 \pm 0.04$ & $P=.03$ \\
MD & $0.7 \pm 0.038$ & $0.49 \pm 0.047$ & $P=.002$ \\
AD & $1.3 \pm 0.06$ & $1.02 \pm 0.05$ & $P<.001$ \\
RD & $0.4 \pm 0.06$ & $0.23 \pm 0.05$ & $P=.002$ \\
\hline
\end{tabular}

${ }^{\mathrm{a}}$ Data are means and SDs.

on the basis of the standard least squares linear regression model and restricted maximum likelihood method (JMP Pro 13.0 software; SAS Institute, Cary, North Carolina). This model was constructed looking at group differences by assuming ROI level and group (non-ZOOM/ZOOM) composition as the fixed effects and subject as the random effect. A $P$ value of .05 was used throughout to determine statistical significance.

\section{Image-Quality Assessment}

Image-quality assessments were performed on raw datasets for each sequence using the SNR. The signal intensities within manually drawn ROIs for each directional diffusion image were calculated and averaged ( $\left.\mu_{\text {DTI_cord }}\right)$. Next, the SNR was computed for each sequence as follows: $\left.\mathrm{SNR}_{\mathrm{DTI}}=\mu_{\text {DTI_cord }} / \sigma_{\text {air }}\right) .{ }^{10}$ The $\mathrm{SD}$ of air was measured from a large area outside the cord and surrounding tissues.

\section{RESULTS}

The mean AD values of the whole cord along the entirety of the cervical spinal cord in the age-matched controls for the proposed sequences were the following: non-ZOOM $=1.36 \pm 0.086 \times$ $10^{-3} \mathrm{~mm}^{2} / \mathrm{s}$ and ZOOM $=0.99 \pm 0.021 \times 10^{-3} \mathrm{~mm}^{2} / \mathrm{s}$, which show a significant decrease using the $\mathrm{ZOOM}$ sequence $(P=.002)$. The mean $\mathrm{RD}$ values were the following: non-ZOOM $=0.42 \pm$
$0.041 \times 10^{-3} \mathrm{~mm}^{2} / \mathrm{s}$ and $\mathrm{ZOOM}=$ $0.24 \pm 0.037 \times 10^{-3} \mathrm{~mm}^{2} / \mathrm{s}$. A statistically significant decrease was shown in $\mathrm{RD}(P<.001)$. The mean FA (non$\mathrm{ZOOM}=0.65 \pm 0.042$ and $\mathrm{ZOOM}=$ $0.74 \pm 0.038$ ) and $\mathrm{MD}$ (non-ZOOM $=$ $0.72 \pm 0.038 \times 10^{-3} \mathrm{~mm}^{2} / \mathrm{s}$ and $\mathrm{ZOOM}=0.50 \pm 0.032 \times 10^{-3} \mathrm{~mm}^{2} / \mathrm{s}$ ) showed a significant increase $(P=.04)$ and decrease $(P=.05)$, respectively, using the ZOOM sequence. Also, these parameters (ie, $\mathrm{FA}, \mathrm{MD}, \mathrm{AD}$, and $\mathrm{RD}$ ) were calculated and compared between proposed sequences in the indirect agematched controls. Statistically significant increases in FA $(P=.03)$ and decreases in $\mathrm{MD}(P=.002), \mathrm{AD}(P<.001)$, and $\mathrm{RD}(P=.002)$ were shown (Tables 1 and 2).

Figures 2 and 3 represent the DTI indices as a function of intervertebral disc levels. In all cases, there is a clear difference between data acquired from the ZOOM and the non-ZOOM sequences. In both direct and indirect age-matched groups, the ZOOM sequence uniformly demonstrated an increase in mean FA values and a decrease in mean $\mathrm{MD}, \mathrm{AD}$, and $\mathrm{RD}$ values at each cervical intervertebral cord level compared with the non-ZOOM method.

Using the spinal cord mask, we calculated means and SDs of

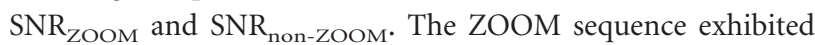
higher SNR $\left(\mathrm{SNR}_{\mathrm{ZOOM}}=22.84 \pm 7.59\right)$ compared with the nonZOOM sequence $\left(\mathrm{SNR}_{\text {non-ZOOM }}=19.7 \pm 7.05\right)$. However, by means of a 2-tailed $t$ test assuming unequal variances, the ZOOM sequence did not demonstrate a statistically significant increase. As seen in Figs 4 and 5, ZOOM DTI shows better definition of WM/GM, CSF, and CSF/cord edge.

\section{Variability of ZOOM EPI versus Full-FOV Single-Shot EPI}

The variability of the DTI values for ZOOM and non-ZOOM sequences was measured using the coefficient of variation $(\mathrm{CoV})$ within direct and indirect age-matched controls. The CoV of the DTI values within direct age-matched subjects showed: $\mathrm{FA}\left(\mathrm{CoV}_{\mathrm{ZOOM}}=\right.$ $\left.0.06 \pm 0.02, \mathrm{CoV}_{\text {non-ZOOM }}=0.1 \pm 0.01\right), \mathrm{MD}\left(\mathrm{CoV}_{\text {ZOOM }}=0.14 \pm\right.$ $\left.0.05, \mathrm{CoV}_{\text {non-ZOOM }}=0.2 \pm 0.05\right), \mathrm{AD}\left(\mathrm{CoV}_{\mathrm{ZOOM}}=0.14 \pm 0.04\right.$, $\left.\mathrm{CoV}_{\text {non-ZOOM }}=0.24 \pm 0.13\right)$, and $\mathrm{RD}\left(\mathrm{CoV}_{\text {ZOOM }}=0.25 \pm 0.06\right.$, $\left.\mathrm{CoV}_{\text {non-ZOOM }}=0.31 \pm 0.09\right)$. Less variability was shown in DTI using the ZOOM sequence compared with the non-ZOOM sequence. Similar results were shown in the indirect age group as follows: FA $\left(\mathrm{CoV}_{\text {ZOOM }}=0.06 \pm 0.01, \mathrm{CoV}_{\text {non-ZOOM }}=0.09 \pm 0.02\right)$, $\mathrm{MD}\left(\mathrm{CoV}_{\text {ZOOM }}=0.18 \pm 0.04, \mathrm{CoV}_{\text {non-ZOOM }}=0.19 \pm 0.04\right), \mathrm{AD}$ $\left(\mathrm{CoV}_{\text {ZOOM }}=0.16 \pm 0.03, \mathrm{CoV}_{\text {non-ZOOM }}=0.22 \pm 0.02\right)$ and $\mathrm{RD}$ $\left(\mathrm{CoV}_{\text {ZOOM }}=0.28 \pm 0.05, \mathrm{CoV}_{\text {non-ZOOM }}=0.23 \pm 0.03\right)$.

\section{DISCUSSION}

This report demonstrates that a ZOOM-EPI DTI sequence acquires more precise high-resolution cervical spinal cord diffusion images compared with the more common non-ZOOM DTI in the healthy adult cervical spinal cord. In the clinical setting, nonZOOM is commonly used to generate DTI due to its rapid acqui- 

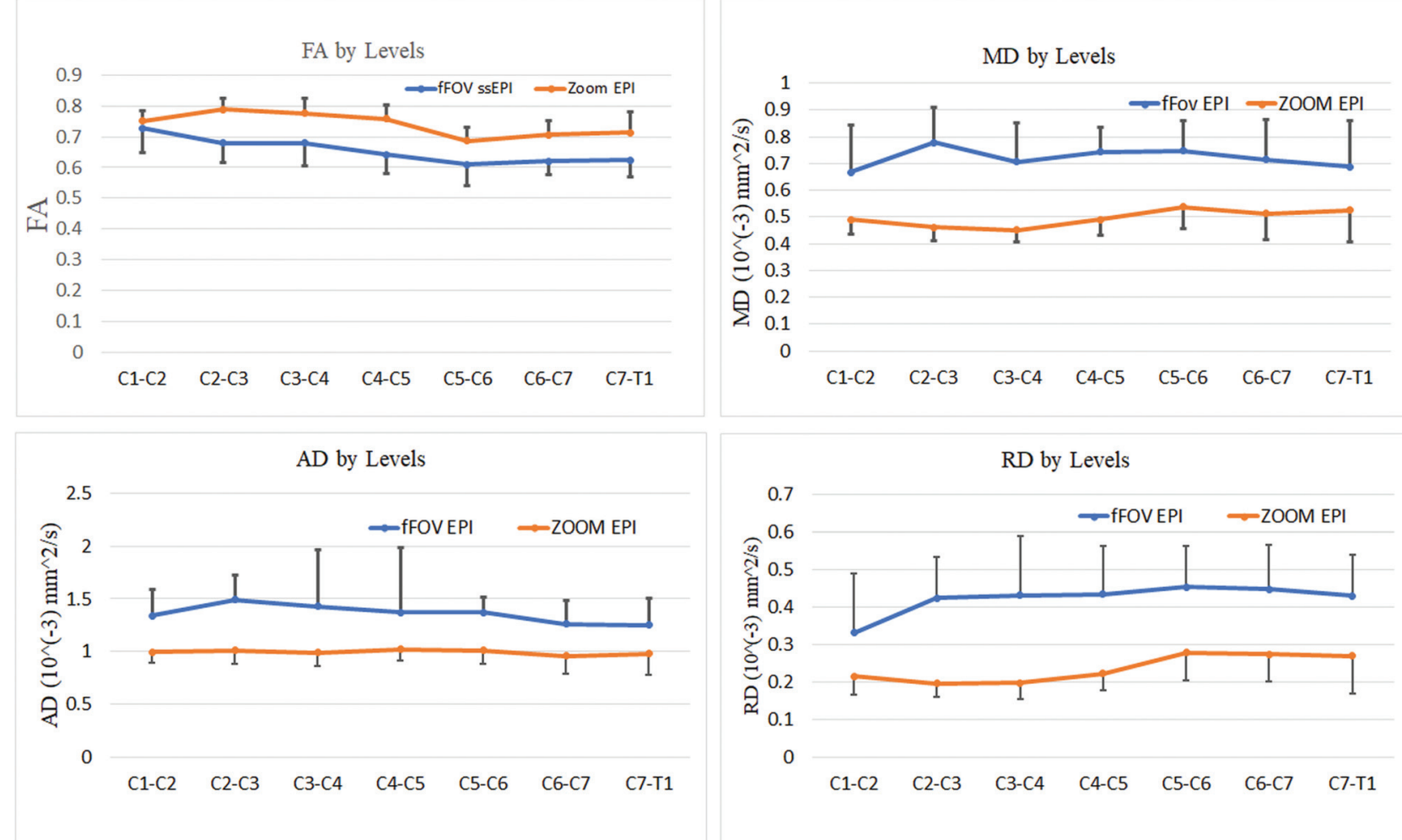

FIG 2. Averaged DTI parameters of ZOOM and non-ZOOM (full-FOV [fFOV] single-shot-EPI [ss-EPI]) sequences for the direct age-matched group as a function of intervertebral disc levels across all subjects. The error bars represent the SDs.
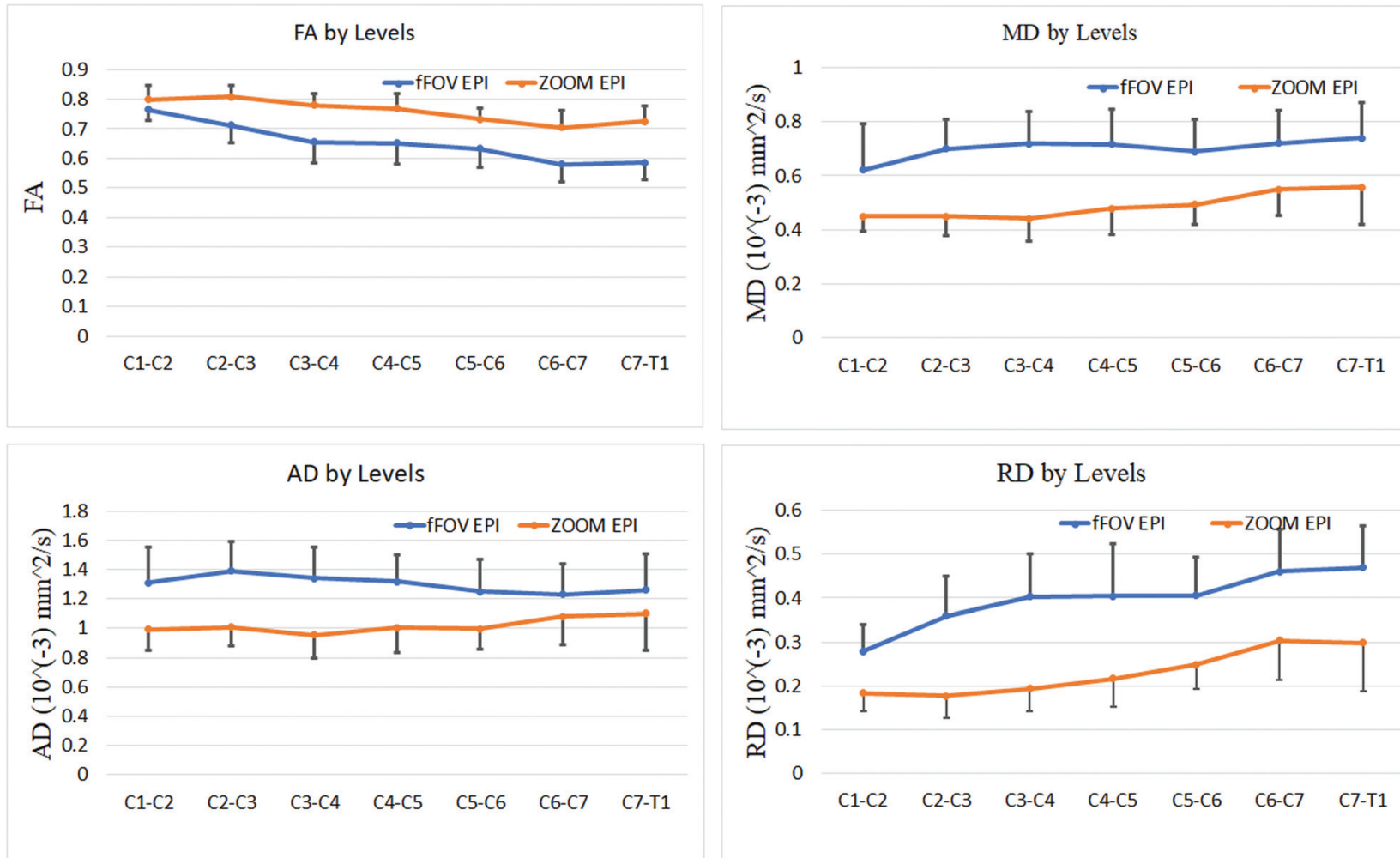

FIG 3. Averaged DTI indices of ZOOM EPI and non-ZOOM (full-FOV [fFOV] single-shot-EPI) sequences for the indirect age-matched group as a function of intervertebral disc levels across all subjects. The error bars represent the SDs. 


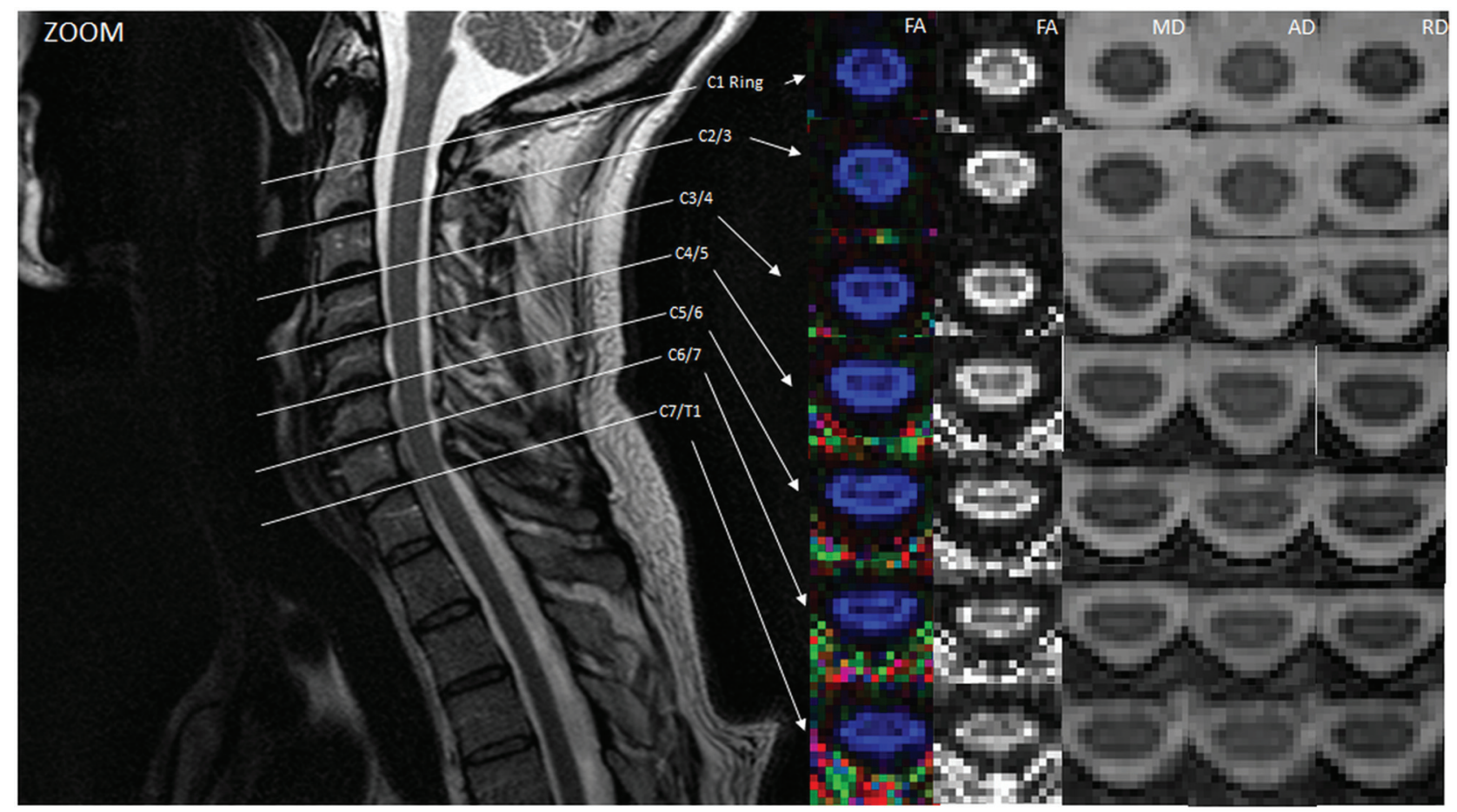

FIG 4. Illustration of DTI indices at different spinal cord levels localized by a T2-weighted image in ZOOM. Midsagittal T2-weighted image shows the location of the 7 axial diffusion tensor images that cover the entire length of the cervical spinal cord. Color and gray-scale FA maps are in the first and second columns. MD, AD, and RD maps at each location are also shown. Note that spatial resolution is improved and distortion is lower compared with the non-ZOOM acquisition (Fig 5). ssEPI indicates single-shot EPI.

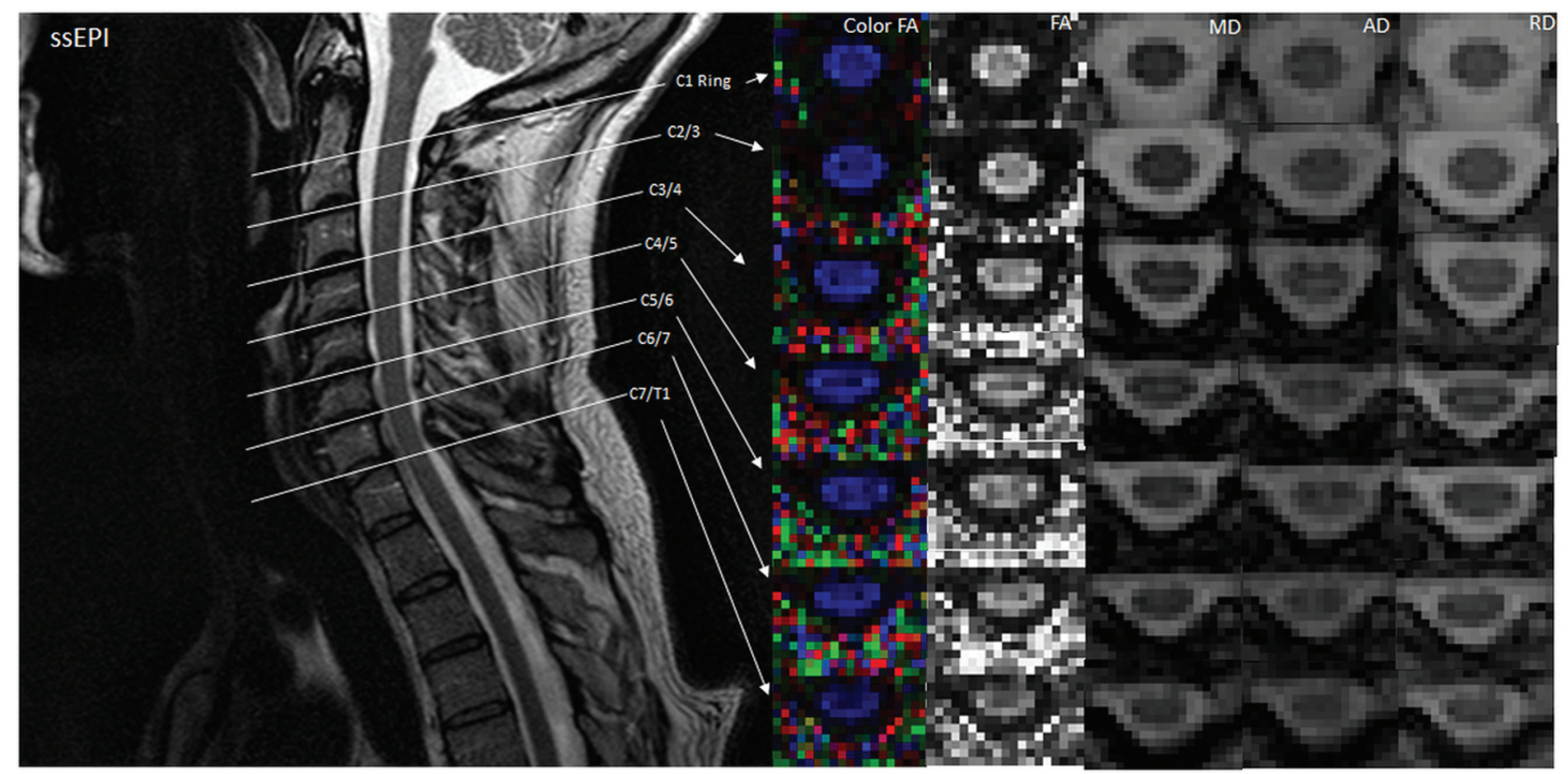

FIG 5. Illustration of DTI indices at different spinal cord levels localized by a T2-weighted image in non-ZOOM. Midsagittal T2-weighted image shows the location of the 7 axial diffusion tensor images that cover the entire length of the cervical spinal cord. Color and gray-scale FA maps are in the first and second columns. MD, AD, and RD maps at each location are also shown.

sition time coupled with a high SNR. These appealing variables result in a quick scan that is relatively insensitive to motion artifacts. However, due to the low pixel bandwidth of EPI along the phase-encoding direction, images are vulnerable to susceptibility artifacts such as chemical shift and geometric distortions. ${ }^{1}$ Additionally, EPI generates poor in-plane spatial resolution. ${ }^{2}$ These disadvantages become especially apparent in smaller anatomic structures like the spinal cord. One way to reduce the probability of these susceptibility artifacts is to limit the FOV along the phase-encoding direction, the method that ZOOM-EPI implements. ${ }^{5,8}$

In the direct age-matched group comparison, the mean FA

AJNR Am J Neuroradiol 39:1555-61 Aug 2018 www.ajnr.org 
values were significantly higher using reduced-FOV ZOOM at each cervical level. $\mathrm{MD}, \mathrm{AD}$, and $\mathrm{RD}$ values were significantly lower at all cervical cord levels compared with the non-ZOOM method. The disparity of these DTI indices generated from these 2 imaging techniques is predominantly a product of the ability of ZOOM to accurately segment white matter from gray matter with artifact reduction. The higher SNR of ZOOM coupled with the manual ROI definition is the significant strengthening factor for the segmentation algorithms in the future.

One limitation of this study was the inability to compare all subjects using a direct age-matched comparison. This would have increased the sample size, allowing greater power to detect differences between the experimental groups and reduce intersubject variability. Although this was not the case, each analysis showed clear differences and patterns, supporting the conclusion that ZOOM offers superior results for DTI acquisitions in the cervical cord.

A major focal point for this study was to develop a protocol appropriate for clinical use. For clinical scans, one must minimize the scan time while maintaining an acceptable image quality. Thus, the number of gradient directions was kept low, resulting in an acquisition time of $<5$ minutes. For future examination of this sequence, additional gradient directions may be considered to further increase image quality with a slight increase in scan time. Past studies have used 15 gradient directions for 7 minutes and 30 seconds ${ }^{11}$ and 20 gradient directions for 8:49 minutes. ${ }^{9}$ Despite the slight increase in scan time, these additional gradient directions have improved DTI metric estimations. Nonetheless, the ZOOM sequence for this study showed clear advantages over conventional single-shot EPI. This limitation could be overcome by imaging the spinal cord using parallel or multiband DTI techniques, which will allow faster imaging.

Several published spinal cord ZOOM studies have evaluated this technique in the thoracic spinal cord using a standard clinical 1.5T scanner. Future exploration of this ZOOM imaging technique will also focus on the general application in the thoracic spinal cord, the intrinsic capability for segmentation and analysis of white matter, and the generation of white matter fiber tracts using tractography. In this evaluation, the ROIs of the spinal cord were manually defined to encompass both gray and white matter in a cross-section of the entire cervical spinal cord. White matter is inherently more anisotropic due to the longitudinal organization of axonal tracts and myelin sheath architecture. ${ }^{12}$ The natively higher resolution and improved SNR capability of ZOOM offer the best means to make this assessment. There are added benefits for ZOOM methods in improved tractography modeling.

The ZOOM technique as implemented on a Philips scanner is based on the technique known as inner volume imaging. Inner volume imaging limits the FOV to acquire the target structure while excluding any extraneous tissues. Like fullFOV, single-shot EPI ZOOM excites a volume using a $90^{\circ}$ radiofrequency pulse followed by a $180^{\circ}$ refocusing pulse. On the contrary, ZOOM uniquely applies this $180^{\circ}$ refocusing pulse obliquely, effectively acquiring a reduced FOV volume of the pertinent tissue.

In general, previous studies have shown that DTI indices obtained using high-field scanners (3T) are statistically significantly different from those generated by $1.5 \mathrm{~T}$ scanners. ${ }^{13,14}$ These quantitative differences are largely a product of the increase in SNR that one can achieve on a $3 \mathrm{~T}$ scanner. In prior studies investigating the effect of field strength on diffusion parameters, there was good agreement, showing a significant increase in FA and a significant decrease in MD. Our results of an increase in FA and a decrease in $\mathrm{MD}, \mathrm{AD}$, and $\mathrm{RD}$ are supported by other literature as well. ${ }^{6,15}$ However, our mean MD values were slightly reduced ${ }^{6,16,17}$ and may due to the slight differences in scanner parameters.

\section{CONCLUSIONS}

The cervical spinal cord data obtained with the ZOOM imaging sequence provide more precise diffusion metrics, $\mathrm{FA}, \mathrm{MD}, \mathrm{AD}$, and $\mathrm{RD}$, with less variability compared with standard full-FOV single-shot EPI. These results suggest that ZOOM DTI is the preferred method for cervical spinal imaging.

\section{REFERENCES}

1. Zaharchuk G, Saritas EU, Andre JB, et al. Reduced field-of-view diffusion imaging of the human spinal cord: comparison with conventional single-shot echo-planar imaging. AJNR Am J Neuroradiol 2011;32:813-20 CrossRef Medline

2. Le Bihan D, Poupon C, Amadon A, et al. Artifacts and pitfalls in diffusion MRI. J Magn Reson Imaging 2006;24:478-88 CrossRef Medline

3. Saksena S, Middleton DM, Krisa L, et al. Diffusion tensor imaging of the normal cervical and thoracic pediatric spinal cord. AJNR AmJ Neuroradiol 2016;37:2150-57 CrossRef Medline

4. Banerjee S, Nishimura DG, Shankaranarayanan A, et al. Reduced field-of-view DWI with robust fat suppression and unrestricted slice coverage using tilted 2D RF excitation. Magn Reson Med 2016; 76:1668-76 CrossRef Medline

5. Thierfelder KM, Scherr MK, Notohamiprodjo M, et al. Diffusionweighted MRI of the prostate: advantages of zoomed EPI with parallel-transmit-accelerated 2D-selective excitation imaging. Eur Radiol 2014;24:3233-41 CrossRef Medline

6. Samson RS, Lévy S, Schneider T, et al. ZOOM or Non-ZOOM? Assessing spinal cord diffusion tensor imaging protocols for multicentre studies. PLoS One 2016;11:e0155557 CrossRef Medline

7. Andre JB, Zaharchuk G, Saritas E, et al. Clinical evaluation of reduced field-of-view diffusion-weighted imaging of the cervical and thoracic spine and spinal cord. AJNR Am J Neuroradiol 2012;33: 1860-66 CrossRef Medline

8. Wheeler-Kingshott CA, Hickman SJ, Parker GJ, et al. Investigating cervical spinal cord structure using axial diffusion tensor imaging. Neuroimage 2002;16:93-102 CrossRef Medline

9. Hodel J, Besson P, Outteryck O, et al. Pulse-triggered DTI sequence with reduced FOV and coronal acquisition at $3 \mathrm{~T}$ for the assessment of the cervical spinal cord in patients with myelitis. AJNR Am J Neuroradiol 2013;34:676-82 CrossRef Medline

10. Polders DL, Leemans A, Hendrikse J, et al. Signal to noise ratio and uncertainty in diffusion tensor imaging at 1.5, 3.0, and 7.0 Tesla. $J$ Magn Reson Imaging 2011;33:1456-63 CrossRef Medline

11. Bosma RL, Stroman PW. Characterization of DTI indices in the cervical, thoracic, and lumbar spinal cord in healthy humans. Radiol Res Pract 2012;2012:143705 CrossRef Medline

12. Wheeler-Kingshott CA, Parker GJ, Symms MR, et al. ADC mapping of the human optic nerve: increased resolution, coverage, and reliability with CSF-suppressed ZOOM-EPI. Magn Reson Med 2002;47: 24-31 CrossRef Medline

13. Huisman TA, Loenneker T, Barta G, et al. Quantitative diffusion tensor MR imaging of the brain: field strength related variance of 
apparent diffusion coefficient (ADC) and fractional anisotropy (FA) scalars. Eur Radiol 2006;16:1651-58 CrossRef Medline

14. Rossi C, Boss A, Lindig TM. Diffusion tensor imaging of the spinal cord at 1.5 and 3.0 Tesla. Rofo 2007;179:219-24 CrossRef Medline

15. Yokohama T, Iwasaki M, Oura D, et al. The reliability of reduced field-of-view DTI for highly accurate quantitative assessment of cervical spinal cord tracts. Magn Reson Med Sci 2018 Mar 23. [Epub ahead of print] CrossRef Medline
16. Dowell NG, Jenkins TM, Ciccarelli O, et al. Contiguous-slice zonally oblique multislice (CO-ZOOM) diffusion tensor imaging: examples of in vivo spinal cord and optic nerve applications. J Magn Reson Imaging 2009;29:454-60 CrossRef Medline

17. Poplawski MM, Gorniak RJ, Dresner MA, et al. Improved reliability of diffusion tensor imaging utilizing reduced field-of-view ZOOMEPI in normal human cervical spinal cord. In: Proceedings of the Annual Meeting of the Radiological Society of North America, Chicago, Illinois. November 30-December 5, 2014 